\title{
Thorough characterization of a Self-Emulsifying Drug Delivery System with Raman hyperspectral imaging: A case study.
}

Pierre-Yves Sacré $^{\mathrm{a}^{*}}$, Lauranne Netchacovitch ${ }^{\mathrm{a}}$, Charlotte De Bleye ${ }^{\mathrm{a}}$, Pierre-François Chavez ${ }^{\mathrm{a}}$, Cécile Servais ${ }^{\mathrm{b}}$, Régis Klinkenberg ${ }^{\mathrm{b}}$, Bruno Streel ${ }^{\mathrm{b}}$, Philippe Hubert ${ }^{\mathrm{a}}$, Eric Ziemons ${ }^{\mathrm{a}}$

\footnotetext{
${ }^{a}$ University of Liege (ULg), Department of Pharmacy, CIRM, Laboratory of Analytical Chemistry, CHU, B36, 4000 Liege, Belgium

${ }^{\mathrm{b}}$ Galephar Research Center M/F, rue du Parc Industriel 39, 6900 Marche en Famenne, Belgium
}

\begin{abstract}
Newly developed drugs often have poor bioavailability due to their poor water solubility (BCS class 2 drugs). It is therefore necessary to develop new strategies to enhance their solubility and their activity, among which, Self-Emulsifying Drug Delivery System (SEDDS). The efficacy of the drugs contained in these preparations is mainly affected by the solid state and the particle size of the active pharmaceutical ingredient (API).

However, it is quite complex, long and expensive to characterize these parameters with classical techniques such as X-Ray powder diffraction, differential scanning calorimetry or hot stage microscopy.

The present article presents, through a case study, the advantages of the Raman hyperspectral imaging in the characterization of such formulations. Indeed, Raman chemical imaging may fully characterize SEDDS with single equipment and operator in a non-destructive way allowing the follow-up of the formulation during stability studies. Raman imaging is therefore a tool of choice in the PAT framework since it increases the knowledge of the formulation and the process.
\end{abstract}


A quantitative multivariate method using Raman hyperspectral imaging to assay the API in the lipid based formulation has been developed and fully validated following the "total error" approach.

\section{Keywords:}

Hyperspectral imaging; Raman spectroscopy; Self-Emulsifying Drug Delivery System; particle size; PAT tool.

*Corresponding Author. Tel.: +32 4366 4324; Fax: +32 43664317

E-mail address: pysacre@ulg.ac.be

Address: Laboratory of Analytical Chemistry, CIRM, Department of Pharmacy, University of Liege, 1 Avenue de l'Hôpital, B36, B-4000 Liege, Belgium 


\section{Introduction}

Following the Biopharmaceutical Classification System (BCS), drugs may be characterized according to their membrane permeability and their aqueous solubility (Amidon et al., 1995). Even if the actual tendency is to produce biopharmaceutical active pharmaceutical ingredient (API), the huge majority of new chemical entities (NCE) are little molecules obtained by organic chemistry synthesis. This implies that most NCE show a poor water solubility (Li et al., 2009) and are classified as BCS class 2 (poor solubility and high permeability). However, the main problem with BCS class 2 drugs is that they exhibit relatively poor bioavailability.

Several strategies may be used to address this: particle size decrease, polymorphism, amorphous drugs, complexation (with cyclodextrins or surfactants), solid solutions and dispersion, soluble prodrugs and salts (Leuner and Dressman, 2000). Another approach is the use of Self-Emulsifying Drug Delivery System (SEDDS). These liquid or semi-solid formulations are encapsulated into hard or soft capsules and generate an emulsion in the gastrointestinal tract facilitating the absorption of the drug (Mullertz et al., 2010). There exist a large variety of excipients that can be used ranging from non-polar lipids to polar lipids. Pouton et al. (Pouton, 2006) proposed a Lipid Formulation Classification System (LFCS) categorizing the lipid based formulations according to their composition. The LFCS classifies the lipid based formulations as Type I if it is composed exclusively of oils, Type II if it is a mix of oils and water-insoluble surfactants, Type IIIA are the formulations composed of mainly of oils and small proportion of water-soluble surfactant, Type IIIB are composed of a small proportion of oils and a majority of water-soluble surfactants and hydrophilic cosolvents and finally Type IV are composed of a mix of surfactants and co-solvents. The studied formulation is categorized as type IV formulation. This type of formulation allows an increased API charge compared to Type I formulations and produces very fine dispersions in an aqueous medium (Mullertz et al., 2010). 
The main parameters that influence the activity of such formulations are the solid-state and the particle size of the API. The solid-state is commonly characterized using differential scanning calorimetry (DSC) (Balakrishnan et al., 2009; Craig, 2006; Kang et al., 2012) or XRay powder diffraction (XRPD) (Docoslis et al., 2007; Hu et al., 2012; Wei et al., 2012) or vibrational spectroscopy (Milović et al., 2012; Nazzal et al., 2002; Stillhart and Kuentz, 2012). However these techniques are costly and require a highly trained user (for XRPD) and are destructive (for DSC).

Particle size analysis of lipid based formulations is a hard task since particles are formed during the cooling of the melt API/excipients matrix. So, it must be performed on the semisolid dosage form after manufacturing. Therefore some researchers rather analyze the particle size of the produced emulsion in an aqueous medium (Agarwal et al., 2009; Ali et al., 2008). However, this is not applicable as quality control of a commercial formulation. Microscopic analysis with scanning or transmission electronic microscopes (SEM or TEM) may also be used (Balakrishnan et al., 2009; Yi et al., 2008a, 2008b; Zhang et al., 2012). But once again it is costly, require highly trained users and it can only be used to see very small surfaces making representativity of the sampling a very big issue. Optical hot stage microscopy (possibly with polarized light) may also be used if the particle size is large enough (Bikiaris et al., 2005; Sprunk et al., 2012). But this technique has many drawbacks such as temperature degradation, polymorphism conversion but most of all the melt down of the smallest particles in the matrix making only possible the observation of the biggest particles. 
Another technique that can be used to characterize both polymorphic state and particle size of the API is the Raman hyperspectral imaging. This technique combines the information obtained by Raman spectroscopy with spatial information.

Three configurations of Raman hyperspectral imaging systems exist: point mapping, line scanning and global imaging. The point scanning mode is by far the most used configuration. It consists of recording a spectrum at a specific spatial location, then the sample moves, another spectrum is recorded at an adjacent location and so on until the whole mapping area is covered. The line scanning mode records the spectra of a complete line of the sampled area simultaneously which fastens the analysis. Finally, in the global imaging configuration, the whole sample is illuminated and the intensity of a fixed number of wavenumbers is recorded returning a complete image of the sample for each wavenumber. In this case, the spatial resolution is limited by the number of pixels of the detector (Sacré et al., 2014a). In the present study, each imaging system worked in the point mapping mode.

Raman spectroscopy allows to characterize the solid-state of pharmaceutical powders (Brittain, 2009; Simone et al., 2014) but also to obtain quantitative information of an API (Breitkreitz et al., 2013) and because of the low wavelength laser used (visible to NIR light), it may provide high resolution $(\sim 1 \mu \mathrm{m})$ images (Adar et al., 2006).

It is therefore possible with a single technique to obtain both solid-state, quantitative and particle size information of a sample. Raman hyperspectral imaging is surely the technique of choice for the characterization of solid dispersions and among them SEDDS.

In the present study, we developed and validated a quantitative method based on hyperspectral Raman imaging to quantify a BCS 2 API in a lipid based formulation. To the author's best knowledge, it is the first time that a hyperspectral imaging quantitative method has been fully validated using the "total error" approach. 
Moreover, two formulations were produced, one with the API totally dissolved in the matrix and a second one with $70 \%$ of the API dissolved in the matrix and $30 \%$ added as bulk powder. The second formulation mimics a bad processed medicine and Raman hyperspectral imaging has been used to characterize both formulations.

\section{Experimental}

\subsection{Samples}

The studied formulation consists of a BCS 2 API dispersed in a lipid matrix at a concentration of $28 \%(\mathrm{w} / \mathrm{w})$. The excipient part is composed of lauroyl macrogol-32 glycerides (>50\%), hydroxypropyl cellulose, macrogol 20000, sodium starch glycolate and ascorbyl palmitate by order of importance.

The API and excipients were kindly donated by Galephar M/F.

\subsubsection{Validation}

Calibration and validation samples were prepared at 50, 75, 100, 125 and $150 \%$ of the target API concentration. Only the ratio API/excipients was changed keeping the ratio between excipients constant. For each concentration level, three independent series were realized with three replicates per series.

The samples were prepared by dispersing the melt API in the melt excipients. Once dispersed, the mix is cooled to room temperature and three small cylindrical samples $(1 \mathrm{~cm}$ of diameter and $0.5 \mathrm{~cm}$ high) are deposited on a microscope slide constituting a series. Samples were then milled with a Leica EM Rapid milling system equipped with a tungsten carbide miller (Leica Microsystems $\mathrm{GmbH}$, Wetzlar, Germany) to prepare their surface for Raman imaging experiments.

After the Raman imaging experiments, samples were assayed by HPLC. 


\subsubsection{Industrial samples}

The validated quantitative method was applied on industrial samples. Three samples per batch and three different batches (517811J, 12F04, 11I19) were analyzed. The semi-solid samples were taken out of the hard capsules and a sagittal cut was performed before milling.

Once mapped, samples were analyzed by HPLC.

\subsubsection{Samples for the case study}

Two preparations were compared and fully characterized using Raman imaging:

- Preparation 1: $100 \%$ of the API has been dissolved in the melted excipients. This preparation corresponds to an industrial batch without manufacturing issue.

- Preparation 2: $70 \%$ of the API has been dissolved in the melted excipients and the remaining $30 \%$ were added as crystalline bulk powder to the solidified cooled preparation. This preparation mimics an industrial batch that encountered issues during process.

\subsection{Reference method}

\subsubsection{Chemicals}

Methanol HPLC grade was purchased from J.T. Baker (Deventer,Netherlands). Water was purified by a Millipore system (18.2 M $/ \mathrm{cm}$ resistivity, Milli-Q) before filtration through a $0.22 \mu \mathrm{m}$ Millipore Millipak ${ }^{\circledR}-40$ disposable filter units (MilliporeCorporation, USA).

\subsubsection{Instrument and chromatographic conditions}

The HPLC experiments were performed on an Alliance 2690 HPLC system (Waters, Milford, USA) coupled to a 2996 PDA detector (Waters). Data acquisition and treatment were realized with the Empower 2.0 software (Waters). 
The analysis was carried out with a LiChrospher ${ }^{\circledR} 100 \mathrm{RP}-8$ column $(125 \times 4 \mathrm{~mm}, 5 \mu \mathrm{m}$; Merck, Darmstad, Germany) kept at $35^{\circ} \mathrm{C}$. Mobile phase consisted of a mixture of $\mathrm{MeOH}$ and water $(80: 20, \mathrm{v} / \mathrm{v})$.

The HPLC system was operated at isocratic mode during 8 min at a flow rate of $0.8 \mathrm{~mL} \mathrm{~min}^{-1}$ and the injected volume was $30 \mu \mathrm{L}$. UV detection was performed at $288 \mathrm{~nm}$.

\subsubsection{Sample preparation}

All dilutions were performed in a mixture of water and $\mathrm{MeOH}(1: 1, \mathrm{v} / \mathrm{v})$ except for the first step performed in $100 \% \mathrm{MeOH}$.

Calibration standards were obtained by successive dilutions of a stock solution of $40 \mathrm{mg}$ of API in $100.0 \mathrm{~mL} \mathrm{MeOH}$ to reach the final concentrations of $40,60,80,100$ and $120 \mu \mathrm{g} \mathrm{mL}^{-1}$. Analysed samples were prepared by diluting $282 \mathrm{mg}$ of semi-solid preparation ( $80 \mathrm{mg}$ of API at the $100 \%$ level) in $100.0 \mathrm{~mL} \mathrm{MeOH}$. This solution is sonicated for 15 minutes at $45^{\circ} \mathrm{C}$, cooled to room temperature and then diluted to reach the final concentration of $80 \mu \mathrm{g} \mathrm{mL}^{-1}$ (at the $100 \%$ level).

Samples concentrations are back-calculated using the linear regression model as response function.

\subsection{Dissolution studies}

Dissolution of the two case study preparations (see 2.1.3.) was performed with a paddle apparatus (European pharmacopoeia apparatus 2). Dissolution profiles were constructed with the mean dissolution values from six dissolution baths for each preparation. Test samples consisted of $675 \mathrm{mg}$ of each preparation. Baths were heated at $37^{\circ} \mathrm{C}$, dissolution medium consisted of pH 5.5 phosphate buffer with $3 \% \mathrm{~m} / \mathrm{v}$ tween 80 and $0.32 \% \mathrm{~m} / \mathrm{v}$ pepsine and the stirring speed was of $100 \mathrm{rpm}$. Five millilitres aliquots were collected from the $900 \mathrm{~mL}$ 
dissolution bath after 30,60,90,120, 150 and 180 minutes. These aliquots were then analyzed by HPLC.

\subsection{Raman hyperspectral imaging analyses}

\subsubsection{Quantitative analyses}

Raman hyperspectral images were collected with a dispersive Raman spectrometer RamanStation 400F (Perkin Elmer, MA, USA) equipped with a two-dimensional CCD detector $(1024 \times 256$ pixel sensor). The laser excitation wavelength used was $785 \mathrm{~nm}$ with a power of $100 \mathrm{~mW}$.

The measured spectral region was $3050-90 \mathrm{~cm}^{-1}$ and the spectral resolution was equal to $2 \mathrm{~cm}^{-}$ ${ }^{1}$. One accumulation with a 1 second exposure time was performed per sample mapping point. The distance between 2 consecutives mapping measurements was fixed at $200 \mu \mathrm{m}$. Background acquisition during mapping was repeated each 20 minutes. The spectra were collected with the Spectrum 6.3.2.0151 (Perkin Elmer) software.

The samples used for the calibration and validation of the quantitative model were mapped over an area of $6 \times 6 \mathrm{~mm}^{2}$ (squares of $30 \times 30$ pixels).

Industrial samples were mapped over an area of $7.6 \times 5 \mathrm{~mm}^{2}$ (rectangles of $38 \times 25$ pixels). Case study samples were mapped over an area of $12 \times 12 \mathrm{~mm}^{2}$ (squares of 60 x 60 pixels).

\subsubsection{Microscopic analyses}

Microscopic imaging analyses of the case study preparations were performed with a Labram HR Evolution (Horiba scientific) equipped with a two-dimensional Newton 970 frontilluminated EMCCD detector $(1600 \times 200$ pixel sensor) (Andor Technology Ltd.), a Leica 50x Fluotar LWD objective and a $785 \mathrm{~nm}$ laser with a power of $45 \mathrm{~mW}$ (XTRA II single frequence diode laser, Toptica Photonics AG). 
A $300 \mathrm{gr} / \mathrm{mm}$ grating fixed at $1200 \mathrm{~cm}^{-1}\left(464-1853 \mathrm{~cm}^{-1}\right)$ was used to perform the mappings with a single acquisition of $0.1 \mathrm{sec}$ (SWIFT TM mode). The confocal slit-hole was fixed at 200 $\mu \mathrm{m}$. A binning factor of 2 and an electron multiplying gain of 150 were applied. The spectra were collected with the LabSpec 6 (Horiba Scientific) software.

One medium resolution mapping of $2500 \times 2500 \mu \mathrm{m}^{2}$ was performed per preparation with a pixel size of $10 \times 10 \mu \mathrm{m}^{2}$ (spatial resolution of $\sim 10 \mu \mathrm{m}$, square mapping of $250 \times 250$ pixels). Three high resolution mappings of $100 \times 100 \mu \mathrm{m}^{2}$ were performed per preparation with a pixel size of $0.5 \times 0.5 \mu \mathrm{m}^{2}$ (resolution of $\sim 1 \mu \mathrm{m}$, square mapping of $200 \times 200$ pixels).

\subsection{Data processing}

Once acquired, the hyperspectral images were unfolded and the spectra were baseline corrected using the Asymmetric Least Squares (AsLS) (Eilers, 2003) algorithm with a $\lambda$ value of $10^{5}$ and a $\mathrm{p}$ value of $10^{-3}$. After baseline correction, cosmic rays have been removed with the algorithm developed by Sabin et al. (Sabin et al., 2012) with a k value of 15 .

The region of interest selection performed with the mapping of the case study samples has been performed with the "htmaskingroipoly" function of the Hypertools toolbox (Amigo, 2010; Vidal and Amigo, 2012).

All computations were realized with Matlab R2013a (version 8.1, The Matworks, Natick, MA, USA).

\subsubsection{Quantitative model}

The average spectrum of each pre-processed calibration mapping was taken to build a PLS regression model. The partial least squares (PLS) analysis of the data has been performed 
with the SIMPLS algorithm (de Jong, 1993) using the PLS Toolbox 7.8 (Eigenvector Research, Wenatchee, WA, USA). Several other approaches were envisaged (median spectrum, ten highest correlated to median spectrum ...) but these approaches gave worse or comparable results and were then abandoned.

The constituted calibration set was standard normal variate (SNV) and mean center corrected before building the model.

A random sample selection cross-validation was performed (10 splits and 10 iterations) to select the number of latent variables to keep. The best model was obtained keeping 2 latent variables with a root mean squared error of cross-validation (RMSECV) of $2.57 \%$.

The validation samples mappings were then analyzed with the PLS model and the mean predicted concentration of each map was used to validate the model.

This method has been validated using the "total error" approach in accordance with the validation requirements in the ICH Q2 R1 norm (Hubert et al., 2008, 2007a, 2007b, 2004). The validation of the assay method has been performed between $50-150 \%$ of the target value. Validation protocol consisted of one operator, one instrument, three replicates per series and three series. Acceptance limits were set at $+/-10 \%$ and the $\beta$-expectation tolerance intervals were computed at a probability $\beta$ of $95 \%$. All validation calculations were performed with Enoval $^{\circledR}$ version 3.0 (Arlenda S.A., Liege, Belgium).

\subsubsection{Particle size analysis}

Medium and high resolution mappings of case study samples were baseline corrected by AsLS ( $\lambda$ value of $10^{5}$ and $\mathrm{p}$ value of $10^{-3}$ ). The baseline corrected mappings were then 
analyzed by multivariate curve analysis - alternating least squares (MCR-ALS) (Jaumot et al., 2005) with two components and with a non-negativity constraint on the spectra and concentration resolution.

The resolved concentration map of the API is then binarized and the particles size estimated. The particle size analysis has been performed using Image J version $1.47 \mathrm{v}$ (Schneider et al., 2012).

\section{Results and discussion}

\subsection{Quantitative method validation}

The quantitative regression PLS model has been fully validated within the $+/-10 \%$ acceptance limits. The classical $+/-5 \%$ acceptance limits for the assay of pharmaceutical specialties were not achievable because of sampling issues. Indeed, only a small slice of the sample is mapped and the representativity of the sample is directly linked to the homogeneity of it.

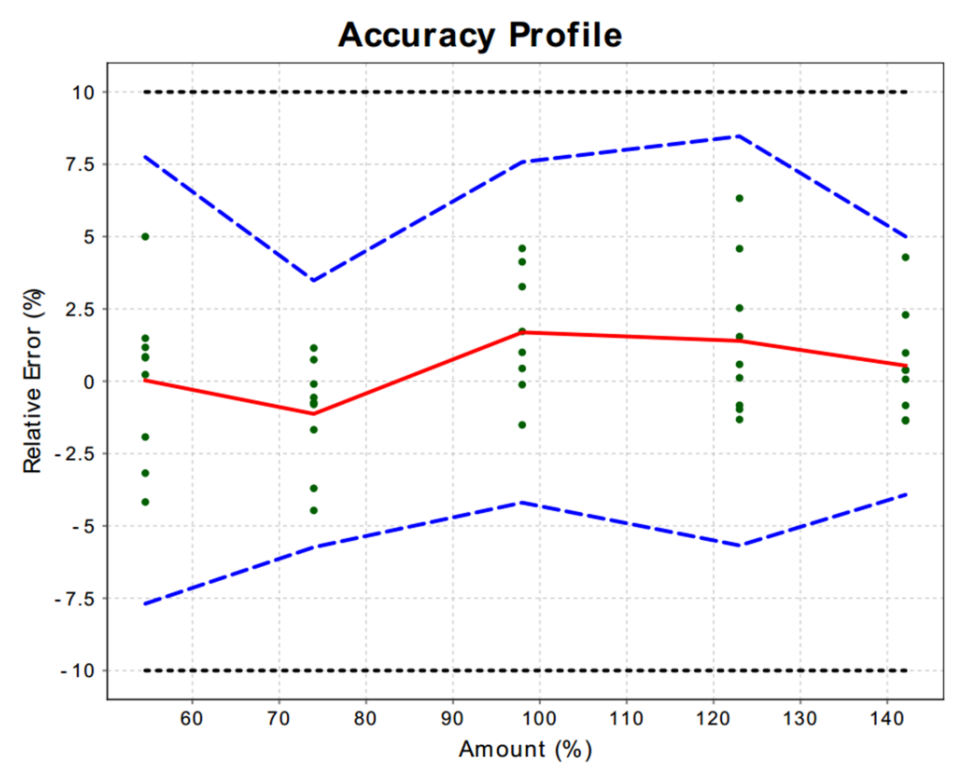

Figure 1: Accuracy profile obtained from the PLS regression model. The solid line is the relative bias, the dashed lines are the $95 \%$-expectation tolerance limits and the dotted lines represent the acceptance limits (+/-10\%). 
Table 1 collects the values of trueness (relative bias), precision (repeatability and intermediate precision) and accuracy (relative $\beta$-expectation tolerance limits) obtained during the validation process. These information may be put together in a single image called accuracy profile (Figure 1) demonstrating that $95 \%$ of future results will fall within the $+/-10 \%$ acceptance limits.

Table 1: Validation criteria of the developed PLS quantitative method.

\begin{tabular}{cccccc}
\hline $\begin{array}{c}\text { Concentration } \\
\text { level } \\
\text { (\% of target } \\
\text { value) }\end{array}$ & $\begin{array}{c}\text { Relative } \\
\text { bias (\%) }\end{array}$ & $\begin{array}{c}\text { Repeatability } \\
\text { (RSD\%) }\end{array}$ & $\begin{array}{c}\text { Intermediate } \\
\text { precision } \\
\text { (RSD \%) }\end{array}$ & $\begin{array}{c}\text { Relative } \boldsymbol{\beta}- \\
\text { expectation } \\
\text { tolerance limits } \\
\text { (\%) }\end{array}$ & $\begin{array}{c}\text { Relative } \\
\text { expanded } \\
\text { uncertainty } \\
\text { (\%) }\end{array}$ \\
\hline $\mathbf{5 0}$ & 0.03 & 2.42 & 2.87 & {$[-7.69 ; 7.75]$} & 6.23 \\
$\mathbf{7 5}$ & -1.13 & 1.88 & 1.88 & {$[-5.74 ; 3.48]$} & 3.97 \\
$\mathbf{1 0 0}$ & 1.69 & 1.99 & 2.21 & {$[-4.20 ; 7.58]$} & 4.77 \\
$\mathbf{1 2 5}$ & 1.40 & 2.41 & 1.72 & {$[-5.68 ; 8.47]$} & 5.85 \\
$\mathbf{1 5 0}$ & 0.54 & 1.82 & 1.82 & {$[-3.93 ; 5.00]$} & 3.85 \\
\hline
\end{tabular}

As one can see, the profile has a small bias but quite large precision values due to the sampling issue. The computed RMSEP value for the validation set was of $2.41 \%$. The accuracy profile demonstrates that the computation of the sole RMSEP does not provide valuable information of the method accuracy (De Bleye et al., 2012).

It is clear that hyperspectral Raman imaging quantitative performances are fairly inferior compared to a classical HPLC method for solid pharmaceutical preparations. But the main advantage of using a validated quantitative model is to obtain reliable quantitative information at the pixel level and therefore more reliable chemical images. Another advantage is the possibility to obtain reliable constitutional homogeneity information with the statistics of the histogram of the pixel concentration values as shown by Breitkreitz et al. (Breitkreitz et al., 2013). 
The developed model was applied on real life industrial samples. Results are shown in Table 2. As one can see, the errors (differences between HPLC and PLS predicted values) are well comprised between the acceptance limits. Beside this quantitative analysis, Raman imaging allows also to obtain information regarding the distributional homogeneity of the API among the sample.

Table 2: Results of the assay of industrial samples by both HPLC and the validated PLS model. Error are expressed as the difference between the PLS and the HPLC assay results.

\begin{tabular}{ccccc}
\hline $\begin{array}{c}\text { Batch } \\
\text { number }\end{array}$ & $\begin{array}{c}\text { Sample } \\
\text { number }\end{array}$ & $\begin{array}{c}\text { HPLC assay } \\
\text { relative } \\
\text { concentration } \\
\mathbf{( \% )}\end{array}$ & $\begin{array}{c}\text { Predicted PLS } \\
\text { relative } \\
\text { concentration (\%) }\end{array}$ & Error (\%) \\
\hline 517811J & 1 & 98,04 & 99,69 & 1,65 \\
& 2 & 98,52 & 96,07 & $-2,45$ \\
& 3 & 97,05 & 93,97 & $-3,08$ \\
12F04 & 1 & 99,23 & 95,61 & $-3,62$ \\
& 2 & 100,03 & 95,41 & $-4,62$ \\
& 3 & 99,13 & 96,28 & $-2,85$ \\
11I19 & 1 & 98,04 & 101,80 & 3,76 \\
& 2 & 98,52 & 104,17 & 5,65 \\
& 3 & 97,05 & 99,99 & 2,94 \\
\hline
\end{tabular}

Figure 2 shows the distribution map of the API in three industrial samples. Both have a correct API predicted content $(98.5 \%, 100.0 \%$ and $104.2 \%$ for sample A, B and C respectively) but they exhibit different distribution pattern. In order to obtain an objective value regarding this difference of distribution, the Distributional Homogeneity Index (DHI) approach was used (Sacré et al., 2014b). The smaller and close to 1 the DHI value, the more homogeneous the sample. Sample A, B and C returned DHI values of 3.53, 8.87 and 4.51 respectively (the number of simulations was set to 100). This result confirms that sample B is the less homogeneous followed by sample $\mathrm{C}$ and sample A. The process should therefore be investigated in order to solve this issue. This might also help choosing the best process 
conditions during formulation development. The DHI values may also be used in a design of experiment as response.

a.

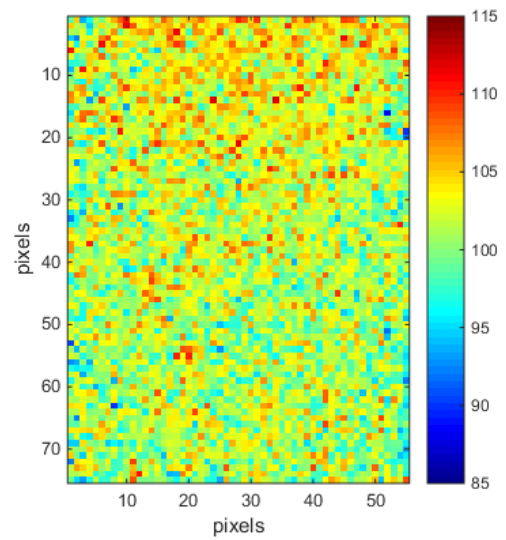

b.

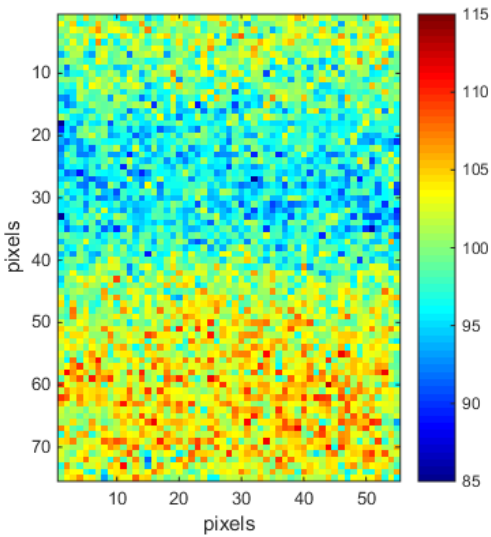

c.

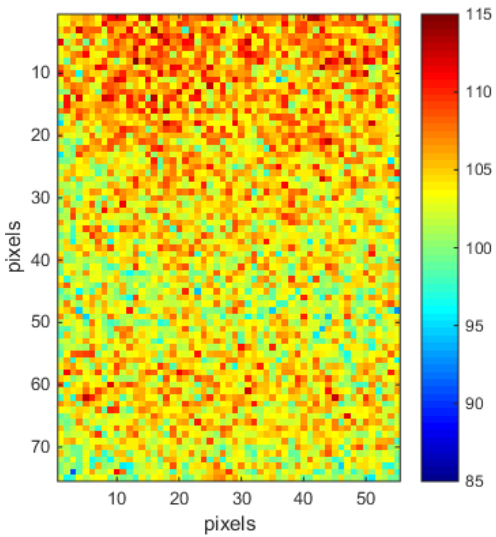

Figure 2:

a. Distribution map of the API obtained applying the developed PLS regression model on an industrial sample (sample A).

b. Distribution map of the API obtained applying the developed PLS regression model on an industrial sample (sample B).

c. Distribution map of the API obtained applying the developed PLS regression model on an industrial sample (sample C).

\subsection{Case Study}

After developing and validating the quantitative model, hyperspectral imaging was used to fully characterize a SEDDS formulation. To illustrate this case study, two preparations were realized: one with the API fully dissolved in the lipidic matrix (preparation 1) and another with $70 \%$ of the API dissolved and $30 \%$ added after cooling of the matrix mimicking a problem in the manufacturing process (preparation 2 ). 
Figure 3 shows the dissolution profiles of both preparations. A difference may be seen since the two curves are parallel. The curve of preparation 2 reaching a maximum of $88.3 \%$ of API dissolved after 180 minutes while preparation 1 reaches $99.4 \%$ at the same time.

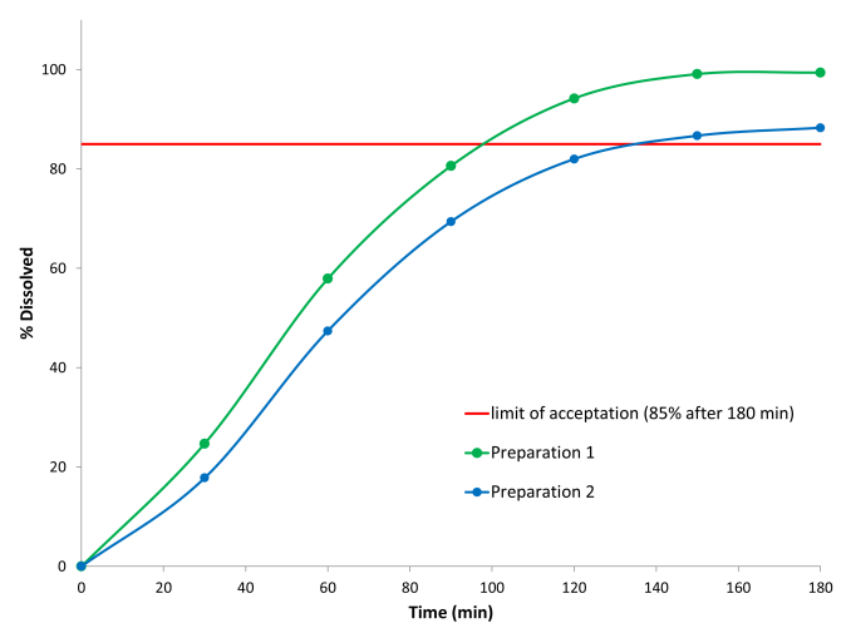

Figure 3: Dissolution profiles obtained for the two case study preparations. Each point is the mean value of six dissolution baths.

Based on these results, three critical parameters such as solid-state, concentration and particle size of the API should be checked. Indeed, it is often expected to be amorphous in order to enhance the API dissolution rate. Figure 4a shows the spectra of the three polymorphic forms of the API while Figure $4 \mathrm{~b}$ shows the spectra extracted from the mapping experiments. As can be seen, the API in both preparations is under its polymorphic 1 (stable) form denoting its crystalline character. It can therefore be concluded that the API is not molecularly dispersed (solid solution) but is present as small crystalline particles dispersed in the excipient matrix. The mean assay values of preparation 1 and 2 are $101.3 \%$ and $101.0 \%$ respectively. The difference between both preparations is therefore neither at the solid-state nor concentration level. 
a.

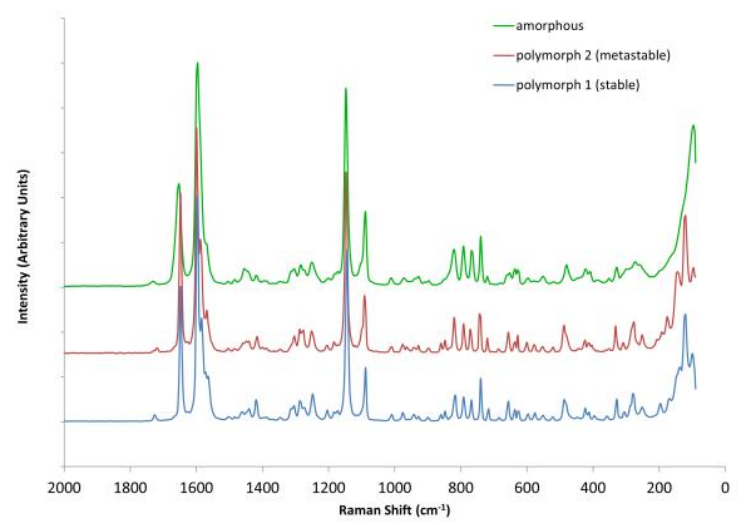

b.

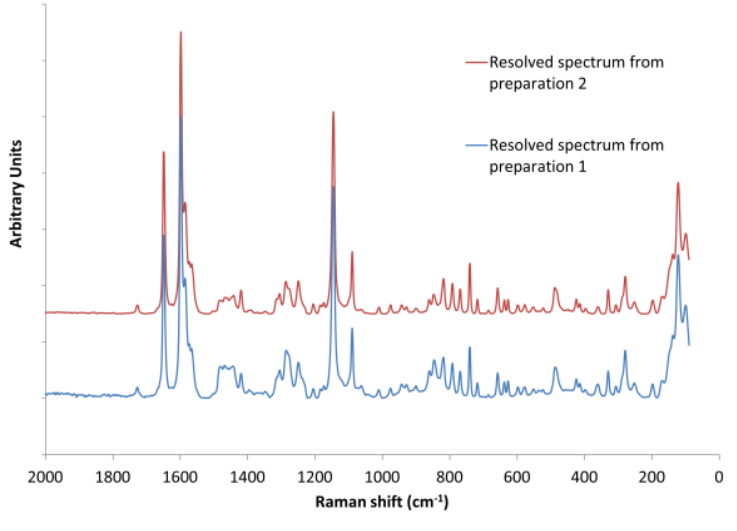

Figure 4:

a. Reference spectra of the different API polymorphic forms.

b. Extracted API spectra from the mapping analysis of preparations 1 and 2.

However, when looking at the constitutional homogeneity, the relative standard deviation among pixels is of $3.3 \%$ and $11.9 \%$ for preparation 1 and 2 respectively. This difference is clearly seen when looking at the Figure $5 \mathrm{a}$ and $\mathrm{b}$. Preparation 1 has a much more homogenous distribution of pixel concentrations while preparation 2 has very concentrated areas and areas with depletion in API.

a.

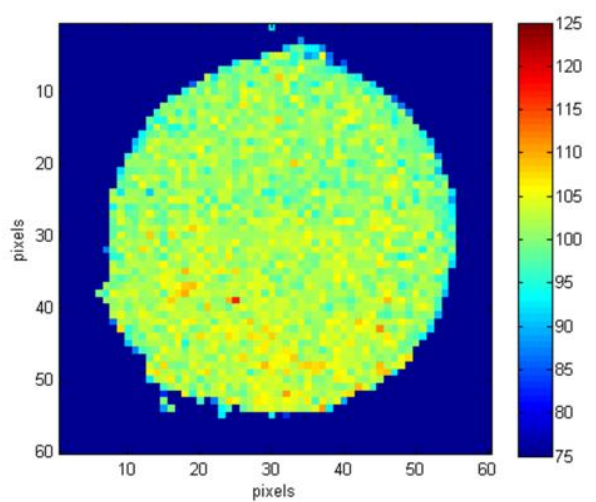

b.

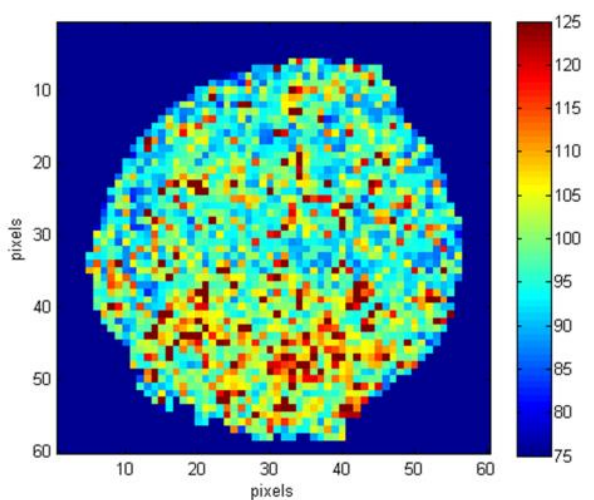

Figure 5: PLS scores for preparation $1(a)$ and $2(b)$ obtained with macroscopic mapping of the dosage forms. 
These results indicate that the major difference between both preparations is situated at the particle size level. Therefore, higher resolution mappings were realized with a confocal microscope to investigate particle sizes up to $1 \mu \mathrm{m}$.

Figures 6 and 7 show the results of the mappings at a medium resolution (pixel size of $10 \mu \mathrm{m}$ ) and high resolution (pixel size of $0.5 \mu \mathrm{m}$ ), respectively. Particles of each mapping have been analyzed and their modal, minimal and maximal diameters of theoretically spherical particles computed. Results are shown in Table 3. Particles of diameter $<15 \mu \mathrm{m}$ and $1.5 \mu \mathrm{m}$ were discarded from the analysis of the medium and high resolution mappings respectively to only take into account reliable size computations. It may be noticed from Figure 6 that a part of the API is also finely distributed at a sub-micron level.

a.

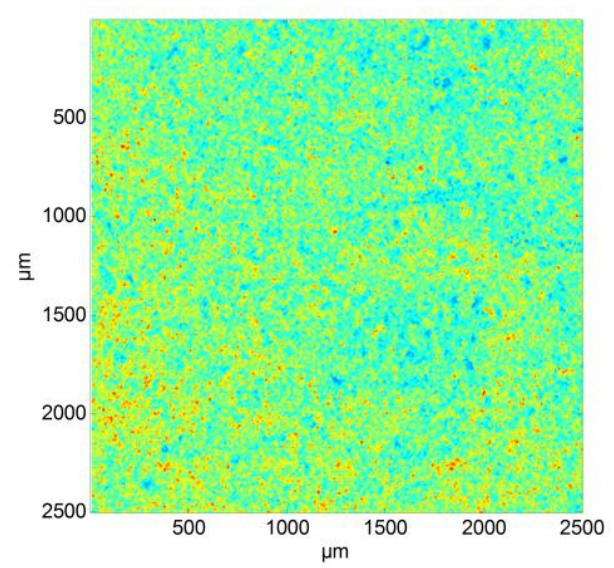

b.

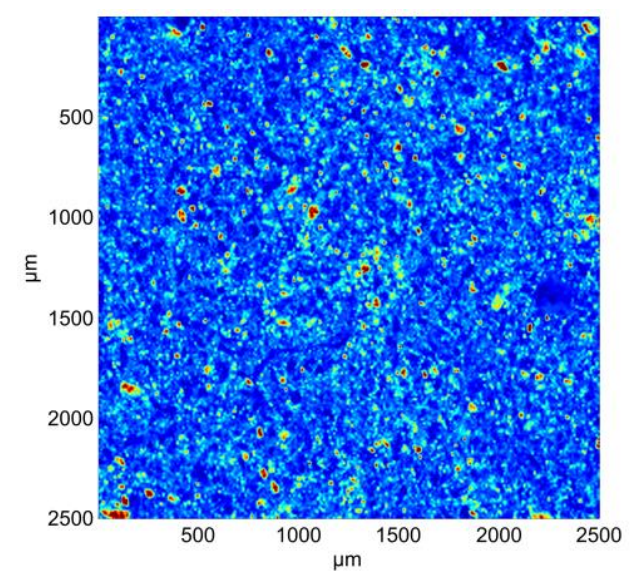

Figure 6: Medium resolution maps of the API obtained by MCR-ALS for the preparation 1 (a) and 2 (b). The pixel size is $10 \mu \mathrm{m}$. 
a.

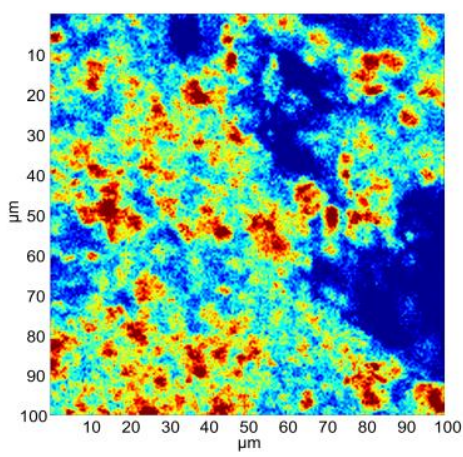

d.

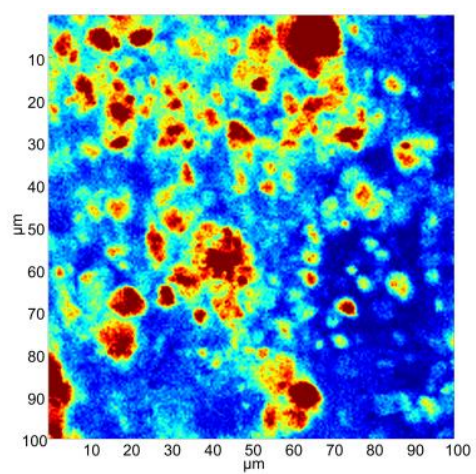

b.

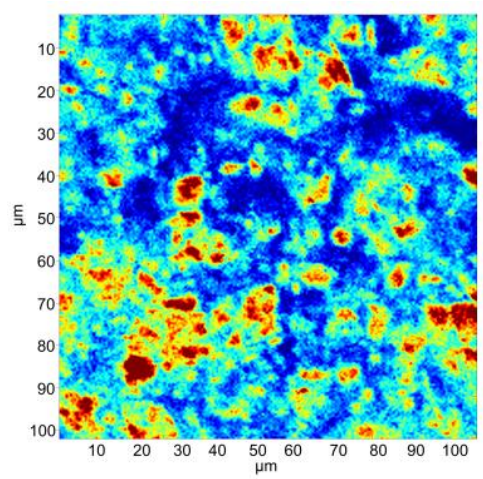

e.

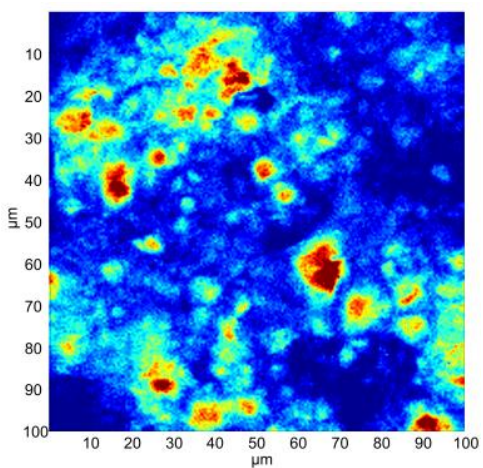

c.

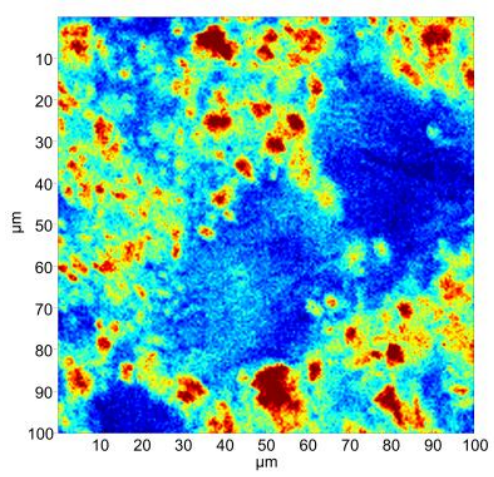

f.

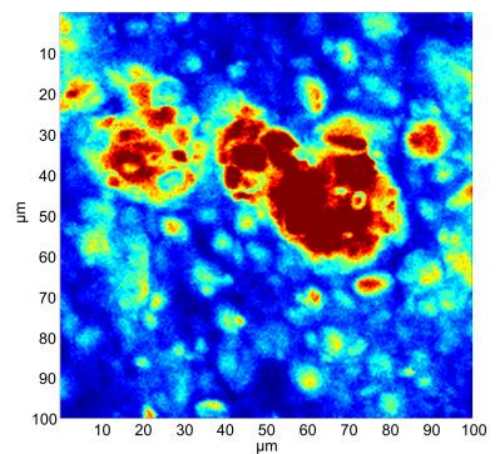

Figure 7: High resolution maps of the API obtained by MCR-ALS for the preparation $1(a, b, c)$ and 2 $(d, e, f)$. The pixel size is $0.5 \mu m$.

Both preparations have approximately the same modal and minimal particle diameter. However, a difference is highlighted in Table 3 regarding the maximal diameter and particularly at the number of particles. Indeed, preparation 1 has a much higher proportion of small particles (567 particles detected in the high resolution mapping versus 19 particles in the medium resolution mapping) than preparation 2 (326 particles detected in the high resolution mapping versus 300 particles in the medium resolution mapping). This confirms that the particle size difference between the two preparations is responsible of the difference in dissolution studies. 
Table 3: Number of particles, modal, minimal and maximal diameter of theoretically spherical particles.

\begin{tabular}{cccccc}
\hline Mappings & Preparation & $\begin{array}{c}\text { Number of } \\
\text { particles }\end{array}$ & $\begin{array}{c}\text { Modal } \\
\text { diameter } \\
(\boldsymbol{\mu m})\end{array}$ & $\begin{array}{c}\text { Minimal } \\
\text { diameter }(\mu \mathrm{m})\end{array}$ & $\begin{array}{c}\text { Maximal } \\
\text { diameter }(\mu \mathrm{m})\end{array}$ \\
\hline $\begin{array}{c}\mathbf{2 5 0 0 \times 2 5 0 0} \\
\boldsymbol{\mu} \mathbf{m}^{2}\end{array}$ & 1 & 19 & 17.6 & 17.2 & 22.8 \\
$\mathbf{1 0 0 \times 1 0 0}$ & 2 & 300 & 17.8 & 17.2 & 188.8 \\
$\boldsymbol{\mu m}^{\mathbf{2}}$ & 2 & 567 & 1.7 & 1.6 & 14.0 \\
\hline
\end{tabular}

It appears from these results that the control of particle size in the final dosage form could be a good quality control test since it is directly related to the bioavailability of the API and therefore to the activity of the drug.

\section{Conclusion}

The present article shows for the first time the validation of a quantitative Raman imaging method following the "total error" concept. Due to the sampling representativeness issue, limits of acceptation were set at $+/-10 \%$. The validated quantitative method allowed obtaining reliable quantitative data per pixel and therefore more representative chemical images of the sample.

A case study with the analysis of two preparations (one with the API fully dissolved and another with the API partially dissolved in the lipidic matrix) showed how Raman chemical imaging analysis may help to characterize lipid based formulations of poorly soluble APIs (BCS class 2). 
Beside these techniques, Raman hyperspectral imaging may be successfully used to fully characterize lipid based formulations. Indeed, with a single equipment, one may obtain quantitative information, know the solid-state form and the particle size distribution of the API in a non-destructive way. This characteristic of Raman spectroscopy is particularly useful for stability studies that can be performed on the same samples all along.

It can therefore be concluded that Raman hyperspectral imaging is the technique of choice for the full characterization of lipid based formulations.

\section{Acknowledgments}

A research grant from the Belgium National Fund for Scientific Research (FNRS) to one of us (C. De Bleye) is gratefully acknowledged. Financial supports from the Walloon Region of Belgium are also gratefully acknowledged for the funding of P.-Y. Sacré and L. Netchacovitch with the convention funds of $\mathrm{N}^{\circ} 1117469$ and $\mathrm{N}^{\circ} 1217716$ respectively. 


\section{$\underline{\text { References }}$}

Adar, F., Lee, E., Mamedov, S., Whitley, A., 2006. Raman Imaging: Defining the Spatial Resolution of the Technology. Spectrosc. Suppl. Raman 38-43.

Agarwal, V., Siddiqui, A., Ali, H., Nazzal, S., 2009. Dissolution and powder flow characterization of solid self-emulsified drug delivery system (SEDDS). Int. J. Pharm. 366, 44-52. doi:10.1016/j.ijpharm.2008.08.046

Ali, H., Nazzal, M., Zaghloul, A.-A.A., Nazzal, S., 2008. Comparison between lipolysis and compendial dissolution as alternative techniques for the in vitro characterization of alpha-tocopherol self-emulsified drug delivery systems (SEDDS). Int. J. Pharm. 352, 104-14. doi:10.1016/j.ijpharm.2007.10.023

Amidon, G., Lennernäs, H., Shah, V., Crison, J., 1995. A theoretical basis for a biopharmaceutic drug classification: the correlation of in vitro drug product dissolution and in vivo bioavailability. Pharm. Res. 12, 413-420.

Amigo, J.M., 2010. Practical issues of hyperspectral imaging analysis of solid dosage forms. Anal Bioanal Chem 398, 93-109. doi:10.1007/s00216-010-3828-z

Balakrishnan, P., Lee, B.-J., Oh, D.H., Kim, J.O., Hong, M.J., Jee, J.-P., Kim, J.A., Yoo, B.K., Woo, J.S., Yong, C.S., Choi, H.-G., 2009. Enhanced oral bioavailability of dexibuprofen by a novel solid Self-emulsifying drug delivery system (SEDDS). Eur. J. Pharm. Biopharm. 72, 539-545. doi:http://dx.doi.org/10.1016/j.ejpb.2009.03.001

Bikiaris, D., Papageorgiou, G.Z., Stergiou, A., Pavlidou, E., Karavas, E., Kanaze, F., Georgarakis, M., 2005. Physicochemical studies on solid dispersions of poorly watersoluble drugs: Evaluation of capabilities and limitations of thermal analysis techniques. Thermochim. Acta 439, 58-67. doi:http://dx.doi.org/10.1016/j.tca.2005.09.011

Breitkreitz, M.C., Sabin, G.P., Polla, G., Poppi, R.J., 2013. Characterization of semi-solid Self-Emulsifying Drug Delivery Systems (SEDDS) of atorvastatin calcium by Raman image spectroscopy and chemometrics. J Pharm Biomed Anal 73, 3-12. doi:10.1016/j.jpba.2012.03.054

Brittain, H., 2009. Vibrational Spectroscopy, in: Brittain, H. (Ed.), Polymorphism in Pharmaceutical Solids. Informa Healthcare, New-York, pp. 347-380.

Craig, D., 2006. Pharmaceutical application of DSC, in: Craig, D., Reading, M. (Eds.), Thermal Analysis of Pharmaceuticals. CRC Press, Boca Raton, pp. 53-96.

De Bleye, C., Chavez, P.F., Mantanus, J., Marini, R., Hubert, P., Rozet, E., Ziemons, E., 2012. Critical review of near-infrared spectroscopic methods validations in pharmaceutical applications. J Pharm Biomed Anal 69, 125-132. doi:10.1016/j.jpba.2012.02.003 
De Jong, S., 1993. SIMPLS: An alternative approach to partial least squares regression. Chemom. Intell. Lab. Syst. 18, 251-263. doi:http://dx.doi.org/10.1016/01697439(93)85002-X

Docoslis, A., Huszarik, K., Papageorgiou, G., Bikiaris, D., Stergiou, A., Georgarakis, E., 2007. Characterization of the distribution, polymorphism, and stability of nimodipine in its solid dispersions in polyethylene glycol by micro-Raman spectroscopy and powder xray diffraction. AAPS J 9, E361-E370. doi:10.1208/aapsj0903043

Eilers, P.H.C., 2003. Parametric Time Warping. Anal Chem 76, 404-411. doi:10.1021/ac034800e

Hu, X., Lin, C., Chen, D., Zhang, J., Liu, Z., Wu, W., Song, H., 2012. Sirolimus solid selfmicroemulsifying pellets: Formulation development, characterization and bioavailability $\begin{array}{lllll}\text { evaluation. Int } & \text { J Pharm }\end{array}$ doi:http://dx.doi.org/10.1016/j.ijpharm.2012.07.055

Hubert, P., Nguyen-Huu, J.J., Boulanger, B., Chapuzet, E., Chiap, P., Cohen, N., Compagnon, P.A., Dewe, W., Feinberg, M., Lallier, M., Laurentie, M., Mercier, N., Muzard, G., Nivet, C., Valat, L., 2004. Harmonization of strategies for the validation of quantitative analytical procedures. A SFSTP proposal--Part I. J Pharm Biomed Anal 36, 579-586. doi:10.1016/j.jpba.2004.07.027

Hubert, P., Nguyen-Huu, J.J., Boulanger, B., Chapuzet, E., Chiap, P., Cohen, N., Compagnon, P.A., Dewe, W., Feinberg, M., Lallier, M., Laurentie, M., Mercier, N., Muzard, G., Nivet, C., Valat, L., Rozet, E., 2007a. Harmonization of strategies for the validation of quantitative analytical procedures. A SFSTP proposal--part II. J Pharm Biomed Anal 45, 70-81. doi:10.1016/j.jpba.2007.06.013

Hubert, P., Nguyen-Huu, J.J., Boulanger, B., Chapuzet, E., Cohen, N., Compagnon, P.A., Dewe, W., Feinberg, M., Laurentie, M., Mercier, N., Muzard, G., Valat, L., Rozet, E., 2007b. Harmonization of strategies for the validation of quantitative analytical procedures. A SFSTP proposal--part III. J Pharm Biomed Anal 45, 82-96. doi:10.1016/j.jpba.2007.06.032

Hubert, P., Nguyen-Huu, J.J., Boulanger, B., Chapuzet, E., Cohen, N., Compagnon, P.A., Dewe, W., Feinberg, M., Laurentie, M., Mercier, N., Muzard, G., Valat, L., Rozet, E., 2008. Harmonization of strategies for the validation of quantitative analytical procedures: a SFSTP proposal part IV. Examples of application. J Pharm Biomed Anal 48, 760-771. doi:10.1016/j.jpba.2008.07.018

Jaumot, J., Gargallo, R., de Juan, A., Tauler, R., 2005. A graphical user-friendly interface for MCR-ALS: a new tool for multivariate curve resolution in MATLAB. Chemom. Intell. Lab. Syst. 76, 101-110. doi:http://dx.doi.org/10.1016/j.chemolab.2004.12.007

Kang, J.H., Oh, D.H., Oh, Y.-K., Yong, C.S., Choi, H.-G., 2012. Effects of solid carriers on the crystalline properties, dissolution and bioavailability of flurbiprofen in solid selfnanoemulsifying drug delivery system (solid SNEDDS). Eur. J. Pharm. Biopharm. 80, 289-297. doi:http://dx.doi.org/10.1016/j.ejpb.2011.11.005 
Leuner, C., Dressman, J., 2000. Improving drug solubility for oral delivery using solid dispersions. Eur. J. Pharm. Biopharm. 50, 47-60. doi:http://dx.doi.org/10.1016/S09396411(00)00076-X

Li, P., Hynes, S.R., Haefele, T.F., Pudipeddi, M., Royce, A.E., Serajuddin, A.T.M., 2009. Development of clinical dosage forms for a poorly water-soluble drug II: Formulation and characterization of a novel solid microemulsion preconcentrate system for oral delivery of a poorly water-soluble drug. J Pharm Sci 98, 1750-1764. doi:10.1002/jps. 21547

Milović, M., Djuriš, J., Djekić, L., Vasiljević, D., Ibrić, S., 2012. Characterization and evaluation of solid self-microemulsifying drug delivery systems with porous carriers as systems for improved carbamazepine release. Int $\mathrm{J}$ Pharm 436, 58-65. doi:http://dx.doi.org/10.1016/j.ijpharm.2012.06.032

Mullertz, A., Ogbonna, A., Ren, S., Rades, T., 2010. New perspectives on lipid and surfactant based drug delivery systems for oral delivery of poorly soluble drugs. J Pharm Pharmacol 62, 1622-1636. doi:10.1111/j.2042-7158.2010.01107.x

Nazzal, S., Smalyukh, I.I., Lavrentovich, O.D., Khan, M.A., 2002. Preparation and in vitro characterization of a eutectic based semisolid self-nanoemulsified drug delivery system (SNEDDS) of ubiquinone: mechanism and progress of emulsion formation. Int J Pharm 235, 247-265. doi:http://dx.doi.org/10.1016/S0378-5173(02)00003-0

Pouton, C.W., 2006. Formulation of poorly water-soluble drugs for oral administration: Physicochemical and physiological issues and the lipid formulation classification system. Eur. J. Pharm. Sci. 29, 278-287. doi:http://dx.doi.org/10.1016/j.ejps.2006.04.016

Sabin, G.P., Souza, A.M. de, Breitkreitz, M.C., Poppi, R.J., 2012. Desenvolvimento de um algoritmo para identificação e correção de spikes em espectroscopia Raman de imagem. Quim. Nova 35, 612-615.

Sacré, P.-Y., De Bleye, C., Chavez, P.-F., Netchacovitch, L., Hubert, P., Ziemons, E., 2014a. Data processing of vibrational chemical imaging for pharmaceutical applications. J. Pharm. Biomed. Anal. 101, 123-140. doi:10.1016/j.jpba.2014.04.012

Sacré, P.-Y., Lebrun, P., Chavez, P.-F., De Bleye, C., Netchacovitch, L., Rozet, E., Klinkenberg, R., Streel, B., Hubert, P., Ziemons, E., 2014b. A new criterion to assess distributional homogeneity in hyperspectral images of solid pharmaceutical dosage forms. Anal. Chim. Acta 818, 7-14. doi:10.1016/j.aca.2014.02.014

Schneider, C.A., Rasband, W.S., Eliceiri, K.W., 2012. NIH Image to ImageJ: 25 years of image analysis. Nat Meth 9, 671-675.

Simone, E., Saleemi, A.N., Nagy, Z.K., 2014. Application of quantitative Raman spectroscopy for the monitoring of polymorphic transformation in crystallization processes using a good calibration practice procedure. Chem. Eng. Res. Des. 92, 594 611. doi:http://dx.doi.org/10.1016/j.cherd.2013.11.004 
Sprunk, A., Strachan, C.J., Graf, A., 2012. Rational formulation development and in vitro assessment of SMEDDS for oral delivery of poorly water soluble drugs. Eur. J. Pharm. Sci. 46, 508-515. doi:http://dx.doi.org/10.1016/j.ejps.2012.04.001

Stillhart, C., Kuentz, M., 2012. Comparison of high-resolution ultrasonic resonator technology and Raman spectroscopy as novel process analytical tools for drug quantification in self-emulsifying drug delivery systems. J. Pharm. Biomed. Anal. 59, 29-37. doi:10.1016/j.jpba.2011.10.018

Vidal, M., Amigo, J.M., 2012. Pre-processing of hyperspectral images. Essential steps before image analysis. Chemom. Intell. Lab. Syst. 117, 138-148. doi:10.1016/j.chemolab.2012.05.009

Wei, Y., Ye, X., Shang, X., Peng, X., Bao, Q., Liu, M., Guo, M., Li, F., 2012. Enhanced oral bioavailability of silybin by a supersaturatable self-emulsifying drug delivery system (SSEDDS). Colloids Surfaces A Physicochem. Eng. Asp. 396, 22-28. doi:http://dx.doi.org/10.1016/j.colsurfa.2011.12.025

Yi, T., Wan, J., Xu, H., Yang, X., 2008a. Controlled poorly soluble drug release from solid self-microemulsifying formulations with high viscosity hydroxypropylmethylcellulose. Eur. J. Pharm. Sci. 34, 274-280. doi:http://dx.doi.org/10.1016/j.ejps.2008.04.010

Yi, T., Wan, J., Xu, H., Yang, X., 2008b. A new solid self-microemulsifying formulation prepared by spray-drying to improve the oral bioavailability of poorly water soluble drugs. Eur. J. Pharm. Biopharm. 70, 439-444. doi:http://dx.doi.org/10.1016/j.ejpb.2008.05.001

Zhang, Y., Wang, R., Wu, J., Shen, Q., 2012. Characterization and evaluation of selfmicroemulsifying sustained-release pellet formulation of puerarin for oral delivery. Int $\mathbf{J}$ Pharm 427, 337-344. doi:http://dx.doi.org/10.1016/j.ijpharm.2012.02.013 